\title{
Load Balancing based on Nodes Distribution in Mobile Sensor Network
}

\author{
Kartinah Zen, Halikul Lenando, Mohamad Nazim Jambli \\ Faculty of Computer Science and Information Technology, Universiti Malaysia Sarawak, Malaysia. \\ \{kartinah, cool,mnjambli\}@fit.unimas.my
}

\begin{abstract}
In a large scale mobile sensor network, some coordinators may exhaust energy earlier if they are connected with excessive number of mobile nodes compared to the others. Balancing the load could stable and prolong the network lifetime, maximizes coordinators capabilities and improves net-work performance. In this paper, a mechanism to balance the coordinators' load based on the distribution of mobile sensor nodes in IEEE 802.15.4 protocol is presented. In this scheme, a coordinator is a static cluster head that collects data from the associated mobile sensor nodes. From the simulation results, the load balancing scheme successfully balances the distribution of mobile sensor nodes that attached to each coordinators. This therefore balances the energy consume among the coordinators and indirectly, delay in data reception is reduced when there are large number of mobile nodes in the system.
\end{abstract}

\section{INTRODUCTION}

Load balancing in wireless sensor network (WSN) improves network stability by distributing the associated nodes as evenly as possible among coordinators. Without load balancing, a coordinator with excessive number of mobile nodes may lead to more energy consumption and experiences data reception delay as compared to the other coordinators. Load balancing maximizes coordinators capabilities and reduces delay in data transmission. In a mobile sensor network (MSN), the frequent changes in large scale network topology lead to a challenge for delay sensitive data reception. When nodes which are associated with the same coordinator send data at the same time, there will be a delay or a failure of data received by the coordinator. This could causes inaccurate information because the sensor node may already move to another location. For example, in an animal monitoring system where sensors are attached to the animals, the movement of these animals may reduce the accuracy of sensors reading regarding the animals' location if the information cannot be sent to the coordinator at the specific time.

There are three important issues when designing a load balancing scheme in MSN. First, it must be established within a short time to adapt with the changes in mobile nodes' locations. Second, in order to save energy, it must be implemented with the smallest number of overheads because it will be triggered regularly, depending on the changes in the topology. And finally, the parameter that triggers the action must relate to the changes in the topology.

The load-balancing schemes in WSN proposed by most of the researchers $[1,2,3]$ require each node to broadcast its position to their neighbours during the network clustering. This will create traffic congestion in large networks, because the neighbours have to keep data of each node. In WSN, this is not practical because sensor nodes have very limited memory. The redundant data received by all nodes lead to more overhead and energy spent within a network. Even though the schemes succeed in balancing the energy among the cluster heads, in the long run, the design exploits more energy consumption causes by the overhead messages.

In [4], the load balancing technique is applied in the static sensor nodes when they are not uniformly distributed and associated with gateways. The key idea is to balance the number of nodes associated with each gateways by arranging the nodes that have more than one possible gateway within reach, to associate with least loaded gateways. The gateways exchange information regarding the communication cost and current load in their cluster. This basic idea is adapted into our proposed load balancing scheme, however, our approach is based on the IEEE 802.15.4 protocol to support the mobility of sensor nodes.

In this paper, the load balancing scheme is achieved by applying it to a cluster topology, therefore mobile nodes in the neighbourhood do not need to broadcast their data to all neighbours, but only to its coordinator. In IEEE 802.15.4 MAC protocol, a method to form a cluster network is based on the ZigBee cluster tree. A large scale network may consist of multiple clusters with one personal area network (PAN) coordinator as a root and several coordinators. Coordinators are the static nodes that can be routers, gateways or sensors with more power, act as the backbone to the network. The examples of mobile sensor application are animal or health monitoring, where sensors are attached to the animals or humans respectively.

\section{Overview: Zigbee Cluster Tree}

In a ZigBee cluster tree topology, a network formation can be divided into two phases, which are network initialization and clustering. In network initialization, the first coordinator is nominated as a PAN coordinator and broadcasts its beacon frames to identify itself to other sensor nodes within its range. Other nodes that are in the coverage range of this coordinator may request to associate to form a virtual cluster in the network clustering. Once associated, the nodes, either a coordinator or a mobile sensor will be added as a child in the PAN coordinator's list. The newly joined node adds the PAN 